\title{
Concretos reciclados, posibilidades de investigación desde el pregrado.
}

\section{Recycled concrete: chances to research in pre graduate.}

\author{
Rivera, Emely ${ }^{1}$ Guerrero, Rachel ${ }^{2}$ Espinoza, Pablo ${ }^{3}$ Millon, Gabriela ${ }^{4} \&$ Áreas Esteven $^{5}$ \\ ${ }^{1-4}$ Universidad Nacional de Ingeniería, Recinto Universitario Simón Bolivar, Managua, Nicaragua \\ 1riveralumbiemely@gmail.com / https://orcid.org/0000-0002-3050-5718 \\ 2alejandraguerrerogarcia@gmail.com / https://orcid.org/0000-0003-4699-5245 \\ 3antoniorubiosud21@gmail.com / https://orcid.org/0000-0003-4973-8180 \\ 4millonlopezg@gmail.com ${ }^{5}$ areassteven99@gmail.com \\ Recibido el 27 de marzo de 2020, aprobado el 26 de junio de 2020
}

RESUMEN | El presente artículo forma parte del proyecto de investigación promovido por la Universidad Nacional de Ingeniería, titulado "Riesgo Urbano: aproximación diagnóstica para la ciudad de Managua" el cual se ha desarrollado desde el 2019 y en el transcurso del 2020. El cemento y la mampostería de bloques huecos son de los materiales más utilizados para la construcción en américa latina, así mismo son de los principales volúmenes de desechos que se generan al acabar la vida útil de un edificio, creando impactos negativos al entorno natural. Pero existen otras alternativas para este tipo de desechos como es la reutilización en sub-base para carreteras y la integración en diseños de mezclas para elementos de concreto, bloques u otro tipo de piezas implementadas en la construcción.

En este artículo se exploran tres investigaciones realizadas respecto al tema y se proponen actividades que favorezcan un estudio en la Universidad Nacional de Ingeniería aplicando pruebas básicas al alcance de la tecnología con que se cuenta, a la vez que se sugieren opciones de usos para las diferentes mezclas de concreto reciclado acorde a su capacidad estructural.

PALABRAS CLAVE | Concreto, Reciclaje, Sostenibilidad, Pulso ultrasónico, Energía incorporada

\begin{abstract}
This article is part of the Investigation project promoted by the Engineering National University, titled as "Urban risk: diagnostic approach for the city of Managua" which has been developed since 2009 and nowadays in course. Cement and masonry of hollow blocks are the most used materials for construction in Latin America, likewise they're the main volumes of waste which are generated by the ending of the useful life of the building creating negative impacts to the environment. But there also exist another choice for these kinds of waste as the reuse in sub-base for highways and the incorporation in mixtures designs for concrete elements, blocks or another type of pieces implemented on the construction.
\end{abstract}


We explore 3 research about concrete topic, and propose activities to do studies in the Engineering national University applying the standard tests whit the technology we have, we suggest choices to use different kinds of mixtures for different products accord his structural strength.

KEYWORD | Concrete, Recycling, Sustainability, Ultrasonic Pulse, Built-in Energy

\section{Introducción}

El proceso constructivo en todo el mundo es una industria constante, con crecimientos firmes en algunos países del mundo. Más de la mitad de la población mundial vive actualmente en ciudades o áreas urbanas (Salvador, 2017). Latinoamérica no es la excepción, y en ese respecto, las construcciones con cemento son posiblemente las más solicitadas, especialmente en elementos como bloques huecos realizados a partir de morteros de cemento y arena.

En Nicaragua la gran mayoría de construcciones (especialmente la tipología habitacional) se realizan utilizando sistemas de mampostería confinada o reforzada, las que hacen uso de bloques a partir de cemento. Existen muchas fábricas productoras de bloques o bloqueras, que funcionan en todo el territorio nicaragüense. El Ministerio de Transporte e Infraestructura - MTI en el 2001 tenía registradas 5 empresas industriales, 41 empresas semi industriales y 29 bloqueras artesanales.

Muchas de las construcciones se ven deterioradas por falta de mantenimiento o también por fenómenos sísmicos que son una constante en la región del Pacífico del país, esto conlleva al abandono o demolición (parcial o total) de los edificios, generando de esta manera desperdicios y escombros que se acumulan en tiraderos de basura o en el peor de los casos en espacios baldíos (públicos o privados) causando deterioro a la imagen urbana y a la salud pública de la población local.

Considerando el ciclo de vida de los edificios que contempla como principales momentos:

- La planificación y diseño

- Construcción

- Uso

- Abandono

- Reutilización (cambio de uso)

- Demolición y reciclaje

Podemos percatarnos que los últimos tres momentos tienen especial importancia en el impacto al medio ambiente, a la vez que son los más significativos para consideraciones de reciclaje y por ende de elaboración de Concretos Reciclados. En este artículo se presentan referencias normativas y experiencia de investigaciones vinculadas al tema y algunas reflexiones, con el fin de alumbrar la posibilidad y factibilidad de realizar desde el pregrado investigaciones de este tipo, este trabajo se abordará en el siguiente orden:

- Desarrollo (Antecedentes/ Nacionales e Internacionales) 
- Iniciativas sobre el reciclaje (Investigación, Legislación, Comercio)

- Definición de estándares o indicadores de trabajo

- Materiales procesamiento y diseño de mezclas

- Estudios experimentales con materiales reciclados

- Ventajas del concreto reciclado

- Recomendaciones metodológicas

- Reflexiones

A continuación se mencionarán algunas experiencias exitosas en estos campos, que dan pauta para nuevos procesos de investigación a partir de diferentes materiales de reciclaje provenientes de la industria de la construcción.

\section{Desarrollo:}

\subsection{Antecedentes}

El grupo de estudiantes de años superiores que hemos trabajado este artículo, paralelamente nos encontramos participando en el proyecto de investigación "Riesgo urbano: aproximación diagnóstica para la ciudad de Managua"' bajo el liderazgo del Arq. Erasmo Aguilar, docente de la Facultad de Arquitectura. Uno de los objetivos específicos es la elaboración de propuestas de nuevos materiales de construcción, lo que de cierta manera se logrará con el fundamento de experiencias previas que se mencionan en el acápite de "Estudios experimentales".

Las posibilidades de uso de los materiales residuales provenientes de procesos constructivos son muy amplias y conllevan una serie de beneficios a nivel económico y ambiental. El interés de poder integrar conocimientos acerca del reciclaje de materiales, con énfasis en el concreto, en estudio experimentales a nivel de pregrado, ha dirigido al estudio y análisis de experiencias de investigación exitosas en el tema. La revisión de la documentación generada a través de los respectivos procesos de investigación se hace en función de identificar, conocer e implementar las referencias normativas, herramientas y procedimientos utilizados, dentro iniciativas de innovación en nuevas propuestas de materiales, reciclaje y disminución del impacto ambiental y los costos energéticos implicados en los procesos constructivos.

Esta experiencia es muy enriquecedora porque ha permitido profundizar en la comprensión de los materiales características y requerimientos que deben cumplir, lo cual que es de vital importancia dentro de la vida profesional, ya que a mayor entendimiento de un elemento hay una mejor aplicación del mismo, mejorando la calidad de las propuesta y proyectos que responden a problemáticas muy marcadas como el tema de déficit de viviendas a nivel cualitativo y cuantitativo de Nicaragua.

\subsubsection{Antecedentes nacionales:}

Los antecedentes directos a esta investigación son dos obras publicadas por la Universidad Nacional de ingeniería:

- Vulnerabilidad estructural en viviendas con mampostería de bloques de cemento (Aguilar, Ruiz, \& Morales, 2017): investigación que estudia el comportamiento estructural presentado por los bloques de cemento producidos por distintas bloqueras artesanales y semi-industriales, con la finalidad de identificar si estos cumplen con las especificaciones establecidas en las normativas nicaragüenses. 
- Investigación científica en arquitectura (Aguilar \& Rosales, 2020): este trabajo, resalta la importancia del desarrollo de actividades investigativas en las instituciones educativas a nivel estudiantil y profesional como parte de la necesidad de desarrollo continuo de nuevos conocimientos aplicables a problemáticas reales, dando un aporte así a la sociedad.

Hay que destacar que en la Facultad de Tecnología de la Construcción de la Universidad Nacional de Ingeniería se han desarrollado trabajos monográficos en los cuales se abordan propuestas de mezclas de concreto estándar. Por otra parte, la línea de construcción forma parte importante en el pensum de la carrera de arquitectura, destacando ejercicios de innovación en nuevas propuestas de materiales y el tema del reciclaje.

\subsubsection{Antecedentes internacionales:}

A nivel internacional existen normativas y referencias desde el diseño, elaboración y curado del concreto, e inclusive en otros países se ha empezado a trabajar reglamentación específica para los materiales reciclados.

Destacamos entre las principales referencias las siguientes:

- Guía para el uso de materiales reciclados en construcción (Ihobe S.A., 2016): esta es una iniciativa del gobierno Vasco para generar un mercado de materiales, con tal fin se generan este tipo de guías técnicas, que promueven la incorporación de materiales reciclados en la construcción.

- Definiciones y construcción de especímenes y cilindros de concreto - ASTM C $\quad 125$. Terminología estándar relacionada con el concreto / ASTM-C-192-81. Método estándar para realización y curado de especímenes de concreto en laboratorio / ASTM-C-31. Prácticas normalizadas para elaborar y curar especímenes de concreto en campo

- Análisis granulométrico - ASTM-C-136-96a, JI S A 1102-2006 (Japón), DIN EN 933-1:1997 (Alemania)

- Densidad de partículas y absorción de agua - ASTM-C-127-88 / JI S A 1110-2006 (Japón) / DIN EN 1097-9:1998 (Alemania)

- Pulso Ultrasónico - ASTM C - 597 Método estándar de evaluación de velocidad pulso a través del concreto.

\section{Iniciativas sobre el reciclaje de materiales de construcción}

Las iniciativas relacionadas al reciclaje de materiales se están desarrollando en distintos ámbitos como investigación, legislación, comercio, etc.

\subsection{Investigación}

Existe una constante búsqueda de mejora de la calidad de los concretos a partir de materiales reciclados. Bloques de cemento y concreto procedente de actividades de demolición son de los materiales más estudiados, también los ladrillos cerámicos, cauchos, plástico e incluso vidrios han sido integrados en los diseños de mezclas de manera parcial o total como agregados.

Dentro de las instituciones más involucradas con la temática podemos mencionar el American Concrete Institute (ACI), quienes crearon el comité 555 "Concrete with Recycled Materials" y han publicado investigaciones acerca del comportamiento y factibilidad de los agregados reciclados en el concreto (Bedoya \& Dzul, 2015, pág. 103). 
A su vez podemos mencionar una serie de países que han llevado a cabo proyectos constructivos aplicando materiales reciclados.

Dichos proyectos abarcan: implementación dentro del hormigón estructural con un porcentaje máximo de reemplazo de 20\%, construcciones de viaductos (Holanda); construcción de viviendas (Alemania); losas de entrepiso (Reino Unido); construcción de carreteras y elaboración de concreto estructural; construcción de calles, carreteras y estructuras de escollera; material de subbase de carreteras (Japón); losas, obras exteriores, concreto estructural del Hong Kong Wetland Park (Hong Kong) (Pérez, 2012, págs. 117-118).

\subsection{Legislación}

Además de mejorar las tecnologías de construcción, estas necesitan de un apoyo una vez se quieran desarrollar los proyectos de este tipo. Este apoyo debe ser proporcionado por las leyes y políticas públicas dirigidas específicamente a la construcción sostenible y aprovechamiento de los residuos generados en obras o en procesos de producción.

Bedoya en su investigación sobre el "El concreto con agregados reciclados como proyecto de sostenibilidad urbana", además de presentar los ensayos realizados a los concretos confeccionados y sus respectivos resultados, realiza una recapitulación de los acuerdos y políticas públicas de la región objeto de estudio. No todos los países se han dado a la tarea de formular dichas políticas y de hecho representan una base imprescindible si queremos que este tipo de iniciativas se materialicen y sean efectivas.

Dentro del contexto nacional, en Nicaragua no se tiene mayor recorrido en el tema del reciclaje en general como estrategia de reducción de impactos al ambiente en su proceso de creación de nuevos materiales para construcción, tampoco tenemos normativas aplicables tanto al proceso de gestión, como captación o tratamiento adecuado del desecho de edificios que dieron su vida útil.

\subsection{Comercio}

Economía circular, es un concepto implementado en la política europea, y se refiere a la necesidad de incorporar e implicar masivamente la cadena de valor de construcción. Esto implica entonces a la introducción de un mercado de materiales reciclados convirtiéndolos de residuos a oportunidades económicas y ambientales.

La Vice consejería de Medio Ambiente del Gobierno Vasco y su sociedad pública Ihobe ${ }^{1}$, apuestan por contribuir desde lo público a la generación de un mercado de materiales reciclados con instrumentos como la compra y contracción pública verde, el desarrollo legislativo y la elaboración de guías técnica... (Ihobe S.A., 2016).

Este tipo de iniciativas son beneficiosas para ambas partes las promotoras y constructoras, el promotor tiene la posibilidad de proyectarse de manera más eficaz en los mercados locales y extranjeros, pero para ello sus productos deben estar sujetos a una serie de normativas y control de calidad, lo que ofrece al constructor y comprador el beneficio de poder adquirir productos certificados, de buen rendimiento y que tienen un valor agregado, ser ambientalmente amigables.

\section{Definición de estándares o indicadores de trabajo}

Los materiales reciclados son evaluados con las normativas implementadas para los materiales de construcción de origen natural. Si el área, objeto de estudio, posee sus propias normativas se aplican las disposiciones locales y se deberán considerar normas de carácter internacional como lo son las normas ASTM, ISO. Algunas veces, las normativas representan una limitante (Pérez, 2012, pág. 118), como es el caso de Japón en donde el material reciclado no cumple con la norma JIS A-5308, dirigida a las especificaciones 
de concreto premezclado, y solo se ha implementado este material como sub-base de carreteras.

\section{Materiales procesamiento y diseño de mezclas}

Los materiales son recolectados de actividades de construcción, demolición o de las fábricas productoras. Posteriormente, el proceso de trituración se realiza con maquinaria especializada, esto para para generar condiciones similares a los procesos productivos de los agregados de carácter natural por si se realiza una comparación en el valor que cuesta por ejemplo una mezcla de concreto de agregados naturales versus una de agregados reciclados (Bedoya \& Dzul, 2015, pág. 105) . También la maquinaria especializada garantiza la obtención de distintas granulometrías, para su posterior clasificación granulométrica y su integración en porcentajes a el diseño de mezclas.

La sustitución de agregados se ha realizados en diversas proporciones. Algunos estudios llegan a sustituir el total de los agregados (Bedoya \& Dzul, 2015); otros trabajan con porcentajes más reservados 0\%, 10\%, 20\%, 30\% (Pérez, 2012), la mayor parte de los concretos estructurales solo integran un porcentaje de agregados reciclados para garantizar la mínima o nula disminución de las propiedades del material.

\section{Estudios experimentales con materiales reciclados para construcción de concreto reciclado}

\subsection{Concreto utilizando triturado de ladrillo (de barro cocido)}

Este residuo tiene un potencial importante para ser reciclado en comparación con otros tipos de residuos inertes, debido a que los residuos de ladrillo permiten la obtención de un material fragmentado que es utilizado como agregado reciclado ampliamente manejado en la industria de la construcción lo cual a su vez ayuda a la preservación de los recursos naturales. Esto motiva a investigar acerca del aprovechamiento y revalorización de estos residuos controlando su producción, convirtiéndolo en materia prima para la fabricación de nuevos materiales, contribuyendo al ahorro de energía y a la creación de sistemas constructivos más eficaces y amigables con el medio ambiente.

\section{- Procedimiento realizado}

Se analizaron las propiedades químicas del ladrillo reciclado, así como las propiedades mecánicas del concreto endurecido (flexión y compresión a los 28 días) que presenta en su composición una sustitución del agregado grueso en porcentajes de $10 \%, 20 \%$ y $30 \%$. Se observa la presencia de 5 compuestos, los cuales se comparan con la norma NTC 3493 «Cenizas volantes y puzolanas naturales, calcinadas o crudas, utilizadas como aditivos minerales en el concreto de cemento Portland», norma la cual se cumple a cabalidad con cada uno de los ítems especificados en ella. Los resultados del ensayo indican la viabilidad de utilizar triturado de ladrillo reciclado como agregado grueso en la elaboración de concreto siempre y cuando este no supere el $30 \%$ del agregado natural grueso.

Se realizaron 3 réplicas para cada mezcla de concreto por lo que se elaboraron 12 probetas circulares para el ensayo de compresión y 12 probetas rectangulares para el ensayo de flexión siguiendo el procedimiento descrito en la norma NTC 1377 «Elaboración y curado de especímenes de concreto para ensayos de laboratorio». Para la elaboración de las mezclas de concreto se emplearon agregados naturales (grava y arena) provenientes de canteras localizadas en cercanías al municipio de Sogamoso, Boyacá.

La granulometría de los agregados naturales y agregado reciclado utilizados en la elaboración de las mezclas de concreto se realizó según la norma NTC 77 - Método de ensayo para análisis por tamizado de agregado y la norma NTC 174 - Especificaciones de agregado para concreto.

\section{- Resultado obtenido}


Con respecto a las propiedades físicas de los agregados, se identificó que el agregado grueso reciclado presenta una densidad relativamente baja y una mayor absorción de agua en comparación con los agregados naturales. La consistencia del concreto fresco se midió luego de dos minutos de la fabricación de la probeta, mediante el método del cono de Abrams. Un concreto con buena manejabilidad debe tener una consistencia entre blanda y fluida, es decir un descenso entre los 5 y $\operatorname{los} 16 \mathrm{~cm}$, la consistencia media del concreto fresco resultó con una relación a/c $=0.5$ fue de $74 \mathrm{~mm}$. En la Resistencia a la compresión se logró demostrar que se es bastante similar a los arrojados por un concreto convencional diseñado para soportar una resistencia de $210.10 \mathrm{Kg} / \mathrm{cm} 2$ (3.000 PSI). En el caso de la absorción del concreto endurecido, es notorio su incremento, lo que indica una mayor permeabilidad al agua.

\subsection{Productos a partir de escombros de concreto y mampostería}

Existe una constante búsqueda de mejora de la calidad de los concretos a partir de los materiales reciclados. Bloques de cemento y concreto procedentes de actividades de demolición son de los materiales más estudiados también los ladrillos cerámicos, cauchos, plásticos y vidrio.

Para la elaboración de un material tan usado como el concreto se requiere de materias primas no renovables, las cuales a su vez generan un impacto ambiental negativo al ser obtenidas principalmente mediante minería a cielo abierto. Por ejemplo, Medellín se ve afectada por la explotación de canteras para la obtención de agregados, teniendo como consecuencia una degradación ambiental de la corteza terrestre urbana.

\section{- Métodos empleados desde la caracterización de las materias primas hasta los ensayos y resultados}

a. Selección de materias primas: para los agregados se escogieron aquellos provenientes de canteras. En los escombros seleccionados y triturados se mantuvo una proporción de $50 \%$ de concreto molido y $50 \%$ de mampostería de ladrillo cerámico con mortero de pega, esto, para el caso del agregado fino.

b. Ensayos de resistencia y durabilidad: se confeccionaron muestras cilíndricas de $10 \mathrm{~cm}$ de diámetro y $20 \mathrm{~cm}$ de altura, que luego fueron sumergidas en un tanque de curado con aguas saturadas de cal. Las muestras se llevan a una prensa hidráulica para determinar su resistencia al esfuerzo de compresión a edades de $3,7,14,28,56$, y 91, días.

c. Obtención de los agregados: se precedió a homologar los agregados reciclados, gruesos y finos, a la distribución arrojada por los agregados naturales de referencia. Una vez distribuidos los pesos por cada tamiz, se procedió a la selección de cantidades para obtener los distintos tipos de agregados para sustitución. En base al porcentaje de agregado fino y grueso sustituido en el diseño de mezcla se dio una nomenclatura, ejemplo de ello es $25-\mathrm{R}$ lo cual significa que se sustituye el $25 \%$ del agregado fino y grueso por material reciclado de la granulometría correspondiente.

A continuación se muestran las diferentes combinaciones de agregados naturales y reciclados para la elaboración de las mezclas con su respectiva nomenclatura:

- Agregado grueso natural.

- Agregado fino natural.

- Agregado grueso 25-R (75\% natural -25\% reciclado)

- Agregado fino $25-\mathrm{R}$ (75\% natural $-25 \%$ reciclado)

- Agregado grueso 50-R (50\% natural -50\% reciclado) 
- Agregado fino 50-R (50\% natural $-50 \%$ reciclado)

- Agregado grueso 100-R (100\% reciclado)

- Agregado fino 100-R (100\% reciclado)

Como punto de referencia para las mezclas que contienen porcentajes de material reciclado, se realizó una mezcla de referencia, la cual fue elaborada en su totalidad (uso del 100\%) con agregados naturales. Ésta fue un punto de comparación y análisis de las muestra generadas con combinación de material natural y reciclado, pudiéndose evaluar si las muestras varían en características, comportamiento y propiedades mecánicas.

\section{- Porosidad}

La mezcla 25-R mantiene un comportamiento casi igual a la mezcla de referencia. Las demás muestras recicladas también presentan un comportamiento muy positivo, como es el caso de la mezcla 50-R que, por ejemplo, en cuanto a la densidad Bulk seca fue de 95.54\%; la mezcla 100-R en este mismo aspecto llego a un $89.73 \%$. En cuanto a los valores de porosidad y absorción del concreto endurecido, todas las mezclas presentaron un comportamiento que se inscribe en los parámetros establecidos por distintos investigadores como D. K. Nekrasov.

Se confirma que los agregados obtenidos del reciclaje de escombros, aunque presentan diferencias en algunas características, pueden ser susceptibles de emplearse como materias primas en un nuevo material para la construcción como el concreto, pues no todas las mezclas se requieren para uso estructural.

\subsection{Concreto elaborado con caucho}

Al pasar de los años se ha generado un gran volumen de neumáticos que ya finalizaron su vida útil y ya no tienen ningún beneficio. Este material es muy difícil de eliminar, pero existen diversos medios para darles utilidad a estos desperdicios, sin embargo para que estos desechos no afecten al ambiente deben ser convertidos a una forma que permita una mejor utilización. El reciclaje parece ser la mejor solución para los desechos de caucho, debido a las ventajas ecológicas y económicas que posee.

Los materiales que se obtienen de los residuos de neumáticos, están constituidos por varios polímeros naturales y sintéticos: Caucho Natural (NR), Estireno Butadieno (SBR), Polibutadieno (BR), Polisoprenos Sinteticos (IR), entre otros, que puede ser usados como parte de los componentes de las capas asfálticas que se usan en la construcción de carreteras, con lo que se consigue disminuir la extracción de áridos en canteras. Al implementar este material a las carreteras las ayuda a ser más seguras. También pueden ser usadas en alfombras, aislantes de vehículos o losetas de goma, materiales de fabricación de tejados, pasos a nivel, cubiertas, masillas, aislantes de vibración, entre otros.

El concreto a pesar de la versatilidad que tiene en la construcción existe la necesidad de mejorar sus propiedades de resistencia tensil, dureza, ductilidad y durabilidad. Al incluir el caucho al concreto este aporta a su durabilidad y elasticidad.

\section{- Técnicas y experimentos}

Para el desarrollo de las investigaciones se prepararon compuestos de concreto tradicional, los cuales están conformados de agregados finos y gruesos, agua y cemento. Se elaboraron mezclas de concreto tradicional y mezclas de concreto-caucho en donde la relación agua-cemento se mantuvo de 0.45 y se sustituyó parte de la arena por caucho, el porcentaje del caucho es del $5 \%$ en peso y los tamaños promedios de las partículas fueron mayores o iguales a 1,19 $\mathrm{mm}$ (grueso) menores a 1,19mm (finos). 
La mezcla se elaboró tomando un diseño de mezcla tradicional, en donde se fijó un valor de asentamiento entre 6 y $10 \mathrm{~cm}$. Adicionalmente, se fijó una resistencia a la compresión de $280 \mathrm{Kg} / \mathrm{cm} 2$ (3982.54 PSI) a los 28 días.

Luego se elaboraron probetas cilíndricas normalizadas y de losas. En las primeras 24 horas las probetas fueron cubiertas para evitar la retracción por perdida de humedad, al terminar estas horas se retiraron de los moldes y se almacenan en el tanque de curado. A los 28 días de curado se determinó la resistencia a la compresión y a la tracción. Los ensayos se realizaron a cuatro cilindros por mezcla y el porcentaje de desviación es menor al 10\%. Por lo tanto, el valor de la desviación no se presenta en las diferentes propiedades analizadas.

Se realizaron las medidas de los tiempos de transición de la onda de pulso ultra sónico a las probetas. Se realizaron 10 mediciones a cada uno utilizando vaselina como agente acoplaste entre los transductores y las probetas.

\section{- Resultados.}

Las partículas finas y gruesas muestran una disminución bastante pronunciada al compararlas con los valores de concreto tradicional siendo un porcentaje de disminución entre el 26 y 36\%. Las partículas con agregado grueso en el compuesto generan un menor valor de resistencia a la compresión, al compararla con las partículas finas. Cuando el tamaño de la partícula es el denominado al azar el valor de esta propiedad para el compuesto de concreto, es ligeramente inferior al del concreto tradicional teniendo una variación del $8 \%$.

Los valores de la resistencia a tracción presentan un comportamiento análogo a la resistencia a la compresión, debido a que el caucho afecta de manera similar a ambas propiedades, siendo las variaciones entre el valor del concreto tradicional, con los diferentes tamaños de partículas de caucho utilizados, azar, fino y grueso, de 5, 33 y $29 \%$, respectivamente.

Este comportamiento de las partículas de caucho fino y grueso se debe a que las partículas de caucho actúan como poros dentro de la mezcla, ellas no incrementan la resistencia del concreto como lo hacen los agregados finos de las pastas ya que el caucho es más elástico que el cemento endurecido. También este comportamiento a la resistencia de tracción y compresión se debe al incremento al contenido del aire.

Las probetas que no contenían caucho hacían un ruido estruendoso, esto se debe a que las que poseen caucho tienen una alta capacidad para absorber energía plástica. Las probetas ensayadas soportaron cargas post-fractura y manifestaron desplazamientos significativos, los cuales son parcialmente recuperables, así la masa de concreto es capaz de soportar cargas, aunque esta esté fracturada.

\section{Ventajas del concreto reciclado}

- Generación de múltiples trabajos de investigación, tanto a nivel de pregrado y posgrado, que incluya el capital humano de las universidades, instituciones gubernamentales y empresa privada.

- Integrar el reciclaje de los desperdicios de construcción dentro de la planificación del ciclo de vida del edificio, permite un manejo más coherente y amigable para la reducción de impactos negativos al entorno natural.

- Utilizar estos materiales ya sea en granulometrías bajas o medias, incluso a nivel de partículas más finas como polvo, permite un ahorro en el consumo de otros materiales y dentro de los requisitos de la energía de procesos (Process Energy Requirement - PER), generando una eficiencia del proceso de fabricación individual (que se implica ganancias en los aspectos de los combustibles que se utilizan en la fabricación de los materiales, la relación materiales vs distancias, etc.). 
- La reutilización de materiales de construcción ahorra comúnmente alrededor del $95 \%$ de energía incorporada que de otro modo se desperdiciaría. Algunos materiales como ladrillos y tejas sufren pérdidas por daños de hasta el 30\% en la reutilización. Ahorro de reciclaje de materiales para el reprocesamiento varía considerablemente con ahorros de hasta un 95\% de aluminio, pero sólo el $20 \%$ para el vidrio (Incorporada, 2014).

- Se abren puertas para otro tipo de mercado de construcción de materiales reciclados, que podría dinamizar la industria de la construcción. Con la inversión y tratamiento adecuado, los productos resultantes de estos procesos podrían generar ganancias económicas a quienes se involucren en estas actividades.

\section{Recomendaciones metodológicas}

Así como se rigen por las normativas de construcción del sector donde se realizan las actividades constructivas, se aplican los mismos ensayos que a cualquier otro material de origen natural, de la misma manera se considera las disposiciones locales si se cuentan con ellas y las internacionales siendo las ASTM las más generalizadas.

A nivel de agregados se realizan las clasificaciónes por granulometría, densidad, absorción, peso volumétrico, análisis químico por el método de difracción de rayos X. La cantidad de estudios aplicados está en dependencia del nivel de control que se quiera tener sobre el material y las posibilidades técnicas con las que se dispone. Cabe destacar que existen más métodos para la calsificación de la granulometría en dependencia del material reciclado que se va a utilizar.

Para el diseño de mezclas para concreto estructural, bloques de cemento, pavimentos y otros productos que incluyan materiales agregados son comunes la aplicación de ensayos de revenimiento mediante el método del cono de Abrams, ensayos de compresión y flexión mediante probetas cilíndricas y vigas simples respectivamente, ensayos de absorción, gravedad específica, pulso ultrasónico por mencionar algunos.

De este análisis de las ventajas y experiencias previas utilizando materiales de escombros u otros en la formulación de concretos reciclados, se propone como paso siguiente la realización de cilindros y muestras a partir de estos materiales con la finalidad de identificar posibles usos en la construcción (sea con propósitos estructurales o no estructurales acorde a los estándares nacionales o internacionales). Se recomienda revisar la norma ASTM-C-136-96.

En principio se debe reciclar y seleccionar el material óptimo para este uso, utilizando un proceso de preselección de la materia prima (escombros de bloques de cemento, paredes de concreto, cerámicos o azulejos, etc.), definiendo al menos dos niveles de granulometría que pueden ser:

Tabla 1. Clasificación granulométrica del agregado reciclado

\begin{tabular}{lllll}
\hline Categoría & $\mathbf{N}^{\circ}$ de Tamiz & Peso unitario & $\begin{array}{l}\text { Dimensión } \\
\text { partícula }\end{array}$ & de \\
\hline $\begin{array}{l}\text { Árido fino } \\
\text { Árido grueso } \\
\text { Cemento }\end{array}$ & No Aplica & & \\
& & & No Aplica & \\
\hline
\end{tabular}

Nota: Elaboración Propia

Para lograr este nivel de grano se requerirá la trituración y tamización del material, posterior el almacenamiento del mismo en recipientes herméticos (baldes u otros similares). La trituración se puede realizar de manera manual (con un mazo y utilizando los equipos de seguridad adecuados). Previo se debe 
seleccionar la proporción que se utilizará para la nueva mezcla (cemento, arena regular, agua, material reciclado).

Las pruebas básicas requeridas aplicar para cilindros y muestras a partir del avance hasta el momento son:

- Compresión simple: Se elaboran probetas y se almacenan por un período de al menos 48 horas, en una temperatura de $28{ }^{\circ} \mathrm{C} \pm 8^{\circ} \mathrm{C}$ y una humedad relativa menor al $80 \%$. Luego se refrentan las probetas con azufre a como lo establece la norma ASTM C 1552. Una vez refrentados se ensayan las probetas alineando el centroide de las probetas con el centro de empuje del plato de apoyo de la máquina de ensayo. Para la aplicación de cargas, se aplica la primera mitad de las cargas según una tasa conveniente de tiempo y la segunda mitad se debe aplicar en un tiempo menor que 2 minutos y mayor o igual a 1 minuto.

- Absorción del agua: Se seleccionan al menos tres muestras, a las cuales se le registra su masa. Posteriormente estos se sumergen en agua a una temperatura controlada de entre $15^{\circ} \mathrm{C} \mathrm{y} 27^{\circ} \mathrm{C}$ durante $24 \mathrm{~h}$. Se pesan las muestras suspendidas con un alambre mientras se encontraban sumergidas, registrando su masa sumergida. Luego se deja drenar las piezas durante 1 minuto con una variación permisible de más-menos 5 segundos, una vez drenados se seca la superficie con un paño húmedo y se mide su masa registrando la masa saturada. Por último se procede a llevar las piezas al horno a secar las muestras en un horno ventilado a una temperatura de $110^{\circ} \mathrm{C}$ con una variación permisible de $\pm 5^{\circ} \mathrm{C}$, por un período de 24 horas y así obtener la masa seca al horno.

- Pulso ultrasónico: se utiliza aparatos que genere y mida el tiempo de la transmisión de una serie de pulsos a una muestra de concreto o un elemento constructivo en obra. La superficie debe de estar libre de polvo, arena y se debe aplicar un gel, grasa o cualquier otro acoplante conveniente; si la superficie es uy áspera se debe limar o desgastar un área con dimensiones lo suficientemente amplias para que se acomoden los transductores, una vez colocados se aplican los pulsos y se mide el tiempo de transmisión y junto a la longitud del elemento que está siendo recorrido por los pulsos, a partir de esos datos se calcula la velocidad de las ondas y utilizando tablas ya establecidas se puede obtener una clasificación de la calidad del elemento evaluado.

Actualmente se está trabajando en el diseño de mezclas y las pruebas técnicas para revisión de las posibilidades que ofrece el concreto reciclado. En un artículo próximo se presentarán los resultados y conclusiones obtenidas de las pruebas técnicas así como los diseños de mezclas realizados en esta investigación.

\section{Reflexiones:}

- De acuerdo a la información estudiada y los resultados obtenidos de esos trabajos, se evidencia que es posible integrar los materiales de construcción reciclados de diferentes orígenes a nuevos proyectos de construcción obteniendo variaciones mínimas en el desempeño mecánico y físico. Esto no se convierte en una norma, ya que por ejemplo algunos materiales de concreto pueden tener deterioro o degradación a sus propiedades mecánicas, debido de reacciones químicas como sulfatos u otros.

- Entre los resultados obtenidos se destacan variaciones que van directamente ligadas la proporción con que se integran los agregados, en proporciones de un $20 \%$ se obtienen capacidades muy similares a las de una mezcla tradicional de prueba (Pérez, 2012); mezclas con un 100\% de sustitución de los materiales de origen natural pueden proporcionar desempeños inferiores al $90 \%$ de la mezcla tradicional de prueba, esto no es del todo negativo, los resultados mostrado por (Bedoya \& Dzul, 2015) presentan resistencias al esfuerzo de compresión que rondan los $210.10 \mathrm{Kg} / \mathrm{cm} 2$ (3000 PSI) una capacidad suficiente para ciertas aplicaciones dentro de la construcción. 
- En uno de los estudios, los resultados reflejan una relación de costos del concreto reciclado y del concreto natural, que en una producción a escala industrial estandarizada de agregados reciclados para un metro cubico con porcentaje de sustitución de agregados reciclado presenta el $65 \%$ del costo del agregado natural, lo que lo convierte en una opción económicamente factible.

- Las universidades (principalmente para las carreras de arquitectura e ingeniería civil) deben realizar esfuerzos y crear el interés sobre el tema del reciclaje de concreto en la búsqueda de promover iniciativas de investigación en los estudiantes de pregrado y alianzas gubernamentales y privadas para la realización de los ensayos pertinentes.

- Es imprescindible que los estudiantes universitarios puedan ampliar puntos de vistas y empezar a reconocer como recursos y materia prima lo que actualmente en nuestro contexto visualizamos como desperdicios, más aún en la actualidad donde todos los esfuerzos se están dirigiendo en pro de conceptos como sustentabilidad y resiliencia.

\section{Referencias}

AGUILAR, E., \& Rosales, B. (2020). Investigación científica en arquitectura. Managua: Universidad Nacional de Ingeniería.

AGUILAR, E., Ruiz, H., \& Morales, F. (2017). Vulnerabilidad Estructural en Viviendas con Mampostería de Bloques de Cemento. Managua: Universidad Nacional de Inegeniería.

ALBANO, C., N., C., Hernández, M., Bravo, A. j., \& Guevara, H. (2008). Estudio de concreto elaborado con caucho reciclado de diferentes tamaños de partículas. Revista de la Facultad de Ingeniería Universidad Central de Venezuela, 1-11.

BEDOYA, C., \& Dzul, L. (2015). El concreto con agregados reciclados como proyecto de sostenibilidad urbana. Ingeniería de Construcción, 99-108.

EOHAVEGUREN, T. (2013). Manual de Diseño de Pavimentos de Adoquines de Hormigón. Chile: Gráfica LOM.

IHOBE S.A. (2016). Guía para el uso de materiales reciclados en construcción. Bilbao: Ihobe S.A.

INCORPORADA, E. (22 de Enero de 2014). Obtenido de Gramas.com.mx: http://gramas.com.mx/2014/01/22/ energia-incorporada/

PÉREZ, Á. (2012). Uso del triturado del ladrillo reciclado como agregado grueso en la elbaoración de concreto. INGENIUM, 116-125.

SALVADOR, R. (8 de Mayo de 2017). www.lavanguardia.com. Obtenido de https://www.lavanguardia.com/ economia/20170506/422335535562/el-mundo-crece-en-las-ciudades.html 